\title{
Immuno-haematologic and virologic responses and predictors of virologic failure in HIV-1 infected adults on first-line antiretroviral therapy in Cameroon
}

Henry D Meriki ${ }^{1,2}$, Kukwah A Tufon', Mbunkah H Afegenwi', Bernard A Nyindem², Pascal N Atanga ${ }^{1,3}$, Damian N Anong ${ }^{1}$, Fidelis Cho-Ngwa ${ }^{1}$ and Theresa Nkuo-Akenji ${ }^{1,4^{*}}$

\begin{abstract}
Background: Contemporary data on the immunologic, haematologic and virologic responses and predictors of virologic failure after initiation of free antiretroviral treatment in Cameroon are needed to evaluate the current treatment-monitoring algorithm and to complement efforts to scale-up and improve on the management of HIV infections.

Methods: This was a cross-sectional study conducted between October 2010 and June 2012. A total of 951 participants aged 18-74 years were recruited from selected approved HIV treatment centres of the Northwest and Southwest regions. This comprised 247 males and 704 females. Demographic, self-reported risk behaviours and socioeconomic data were obtained using a structured questionnaire. Full blood and CD4+T-cell counts were done using standard automated techniques. Determination of viral load (VL) was done using Abbott

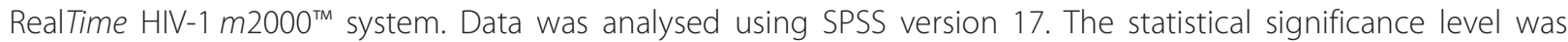
$\mathrm{P}<0.05$.

Results: The median duration of antiretroviral therapy (ART) was 24 months. The population mean CD4 + T-cell count was 255.3 cells/ $\mu \mathrm{L}$ [95\% Cl, $236.8-273.9]$. Overall, $45.9 \%, 43.8 \%$ and $10.2 \%$ of the participants had CD $4+$ T-cell counts of $<200$ cells $/ \mu \mathrm{L}, 200-499$ cells $/ \mu \mathrm{L}$ and $>500$ cells/ $\mu \mathrm{L}$ respectively. Anaemia was present in $26.2 \%$ of the participants with $62.3 \%, 25.7 \%$ and $12 \%$ described as mild, moderate and severe anaemia respectively. Virologic failure occurred in $23.2 \%$ of the participants with $12.3 \%$ having $V L>10,000$ RNA copies $/ \mathrm{mL}$. Meanwhile $76.8 \%$ of patients attained adequate viral suppression with $40.8 \%$ having undetectable viral load. The age group 18-29 years $(p=0.024)$, co-infection with tuberculosis $(p=0.014)$, anaemia $(p=0.028)$ and distance from the treatment centre $(p=0.011)$ independently predicted virologic failure.

Conclusion: The majority of the participants achieved adequate viral suppression after $\geq 6$ months of ART. Despite these favourable immuno-haematologic and virologic outcomes, the National AIDS Control Program should step-up efforts to improve on antiretroviral drug distribution, as well as proper assessment and management of anaemia, foster early diagnosis and treatment of tuberculosis and enhance treatment adherence counselling especially in younger patients.
\end{abstract}

Keywords: Immuno-haematologic, Predictors, Virologic failure, Antiretroviral therapy

\footnotetext{
* Correspondence: wifon@yahoo.com

'Department of Microbiology and Parasitology, University of Buea, P.O. Box 63, Buea, Cameroon

${ }^{4}$ Faculty of Science Clinical Diagnostic Laboratory, University of Buea, Buea, Cameroon

Full list of author information is available at the end of the article
} 


\section{Multilingual abstracts}

Please see Additional file 1 for translations of the abstract into the six official working languages of the United Nations.

\section{Background}

The introduction of free antiretroviral therapy (ART) has substantially improved on the health status of HIV infected patients. Although high levels of adherence have been reported in small-scale HIV programs in Sub-Saharan Africa [1], more challenges arise as these programs scale-up particularly in countries with a growing burden of HIV and tuberculosis (TB), and limited healthcare management facilities [2]. Rapid scale-up of antiretroviral therapy is accompanied by an increasing risk of ART failure resulting from HIV drug resistance and this is a major obstacle to successful ART in HIV-infected patients [3]. ART failure may result in progression to AIDS characterized by immunological and haematological complications and opportunistic infections [4] with increased risk of morbidity and mortality.

Generally, clinical response to ART in resource-limited settings is monitored with CD4 + T cell counts and some hematologic indices [2,5]. For successful outcomes and the amelioration of ARV treatment, the challenge is the effective delivery of ART with the aim of attaining high treatment success rates [5]. However, the assessment of viraemia, which gives a glaring picture of disease progression as well as response to ART [6], is not part of this clinical monitoring in our setting due to its unavailability and cost [2]. With appropriate adherence to ART, it is expected that viral load drops to undetectable levels after $\geq 6$ months of ART [7], which greatly reduces the likelihood of sexual transmission [8], morbidity and mortality among HIV infected patients. Therefore, identifying risk factors predicting treatment outcomes would be a reliable alternative for achieving a high treatment success rate. These identified risk factors could be useful for rendering effective support and services to patients at risk of treatment failure before or during treatment.

HIV prevalence among adults between the ages of 15 and 49 years in Cameroon is $4.3 \%$ [9]. The Northwest $(\mathrm{NW})$ and the Southwest $(\mathrm{SW})$ regions with over 1.8 million and 1.3 million inhabitants respectively [10] shoulder the highest burden of HIV in the country with prevalence of $6.3 \%$ and $5.7 \%$ respectively [9]. The prevalence of HIV in the NW region has been steadily high, even though it declined from $8.5 \%$ recorded in 2010 [11]. The NW and SW regions have 16 and 15 approved treatment centres respectively that take care of over 16,000 and 12,000 patients on ART respectively [11]. Therefore, contemporary data on immunologic, haematologic and virologic responses after the initiation of free antiretroviral therapy in Cameroon are needed to inform and complement efforts to scale-up and improve on the management of HIV infection. In this study, we assess the immunohaematologic and virologic status as well as factors associated with virologic failure of HIV-1 infected patients on ART in NW and SW regions of Cameroon.

\section{Methods}

This was a cross-sectional, hospital-based study involving HIV-infected patients enrolled on high active antiretroviral therapy (HAART), treated between October 2010 and June 2012. Participants were consecutively recruited from the approved HIV treatment Centres of Buea and Limbe Regional hospitals, the management units of the Tiko Central Clinic and Kumba District hospital of the Southwest region; the HIV treatment centre of the Bamenda Regional hospital and the management unit of St. Theresa Catholic Medical Centre of the Northwest region. A nurse assigned to the study obtained demographic and self-reported risk behaviour and socioeconomic data with a standardized questionnaire [12] after obtaining participant's consent.

Two blood samples $(2 \times 5 \mathrm{~mL})$ were collected in ethylene diamine tetra-acetate vaccutainer tubes by a technician from each participant. One tube was centrifuged (at $1100 \mathrm{~g}$ for 20 minutes) and plasma aliquoted and stored at $-20^{\circ} \mathrm{C}$ until needed. The second sample was used for full blood count analysis using the auto haematology analyser (BC-2800, Mindray Bio-Medical Electronics, Shenzhen, China) and CD4 + T-cell count (BD Biosciences FACSCount $^{\mathrm{Tm}}$, New Jersey, USA) following manufacturers' instructions. Anaemia was defined as haemoglobin concentration $<11 \mathrm{~g} / \mathrm{dL}$ [13]. Anaemia was further categorised as mild $(9.6-10.9 \mathrm{~g} / \mathrm{dL})$, moderate $(8-9.5 \mathrm{~g} / \mathrm{dL})$ and severe $(<8 \mathrm{~g} / \mathrm{dL})$. Plasma viral load $(\mathrm{VL})$ was determined using the Abbott RealTime HIV-1 m2000 ${ }^{\text {rm }}$ System (Abbott Molecular Inc. Des Plaines, Illinois, USA) according to the manufacturer's instructions. Virologic failure was defined as VL $>400$ RNA copies/mL after $\geq 6$ months of ART while adequate suppression was VL $\leq 400$ RNA copies/mL after $\geq$ 6 months of ART [14].

The data was analysed with SPSS 17.0 (Statistical Package for the Social Sciences, Chicago, Illinois). Continuous variables were compared using the $t$-test. Univariate analysis was performed with Chi-square and only significant associated risk factors were included in a logistic regression model. Odds ratios (ORs) and nominal 95\% confidence intervals (CIs) were presented. A two-sided p-value $<0.05$ was considered significant for all analyses. Ethical clearance for the study was obtained from Cameroon National Ethics Committee.

\section{Results}

\section{Description of the study population}

Of the 951 participants recruited for the study, 247 were males [mean age: 42.9 years, 95\% CI 41.6 - 44.1] and 
704 were females [mean age: 38.1 years, 95\% CI, $37.4-$ 38.9]. Of 951 these participants, 163 were from the NW and 788 from the SW regions. Over $50 \%$ of the study participants were between the ages of 30 and 45 years old. The majority $(83.6 \%)$ of the study participants had a monthly income of $\leq 50,000$ XAF ( $\leq 100$ US dollars), $48.9 \%$ had attained more than 7 years of education, while $41.7 \%$ reported to be currently married.

Most of the participants $(88.3 \%)$ were receiving treatment at approved treatment centres while $11.7 \%$ were treated at the approved management units. The median duration of ART was 24 months [Range: 1-156 months]. Most of the participants (72.9\%) were on nevirapine (NVP)-based regimen $(17.5 \%$ on $\mathrm{AZT} / 3 \mathrm{TC} / \mathrm{NVP}$ and $55.4 \%$ on $\mathrm{d} 4 \mathrm{~T} / 3 \mathrm{TC} / \mathrm{NVP}$ ) while $27.1 \%$ were on efavirenz (EFV)-based regimen (12.6\% on $\mathrm{AZT} / 3 \mathrm{TC} / \mathrm{EFV}$, and $14.5 \%$ on $\mathrm{d} 4 \mathrm{~T} / 3 \mathrm{TC} / \mathrm{EFV})$. The prevalence of smoking and alcohol consumption in the study population was 9.1\% and $54.3 \%$ respectively. Of the alcohol consumers, $48.2 \%$ were hazardous drinkers (male $>3$ bottles of beer/ occasion and females $>2$ bottles of beer/occasion), while $16 \%$ of the participants both smoked and drank alcohol. Smoking $(p<0.001)$ and alcohol consumption $(p=0.003)$ were significantly higher among males than female participants. A total $10.8 \%$ of the study participants were coinfected with tuberculosis (Table 1).

\section{Immunologic responses}

CD4 + T-cell count measurements were performed in 479 of the 951 participants. The population mean CD4-T-cell count was 255.3 cells $/ \mu \mathrm{L}$ [95\% CI, 236.8 - 273.9]. Mean CD4-T-cell count was significantly higher $(\mathrm{p}<0.001)$ in participants from the SW than NW regions and in the age group $>45$ years when compared with those younger $(\mathrm{p}=0.018)$ (Table 2). This was also true for HIV monoinfected $(\mathrm{p}<0.001)$ when compared with HIV/TB co-infected patients. However, there was no significant difference $(\mathrm{p}=0.171)$ in the mean CD $4+\mathrm{T}$-cell count in patients who were treated with Efavirenz-based and Nevirapinebased regimens. Similarly, CD4 + T-cell count was not significantly different $(\mathrm{p}=0.397)$ between males and females (Table 3). Overall, 45.9\%, 43.8\% and $10.2 \%$ of the participants had CD $4+$ T-cell counts of $<200$ cells $/ \mu L, 200-499$ cells $/ \mu \mathrm{L}$ and $>500$ cells $/ \mu \mathrm{L}$ respectively. CD $4+\mathrm{T}$-cell counts of $<200$ cells $/ \mu \mathrm{L}$ were significantly higher in HIV/ TB co-infected cases $(\mathrm{p}<0.001)$ and in patients from the NW region $(\mathrm{p}=0.002)$ (Figure 1$)$.

\section{Haematologic responses}

A significant difference in haematological parameters for participants from the two regions was observed only in red blood cell $(\mathrm{RBC})$ counts $(\mathrm{p}<0.001)$. Gender had a significant influence $(\mathrm{p}<0.02)$ on mean haemoglobin $(\mathrm{Hgb})$, $\mathrm{RBC}$ and platelet (PLT) counts except white blood cell
(WBC) count (Table 2). HIV-mono infected patients had a significantly higher level of WBC $(\mathrm{p}=0.004)$, PLT $(\mathrm{p}=0.035), \mathrm{RBC}$ counts and Hgb $(\mathrm{p}<0.001)$ when compared with HIV/TB co-infected counterparts. On the other hand, there was no significant difference in most haematologic parameters between EFV-based and NVPbased regimens except for an increase in platelet count levels which was significantly higher $(\mathrm{p}=0.024)$ among patients on EFV-based regimen (Table 3). In general, the prevalence of anaemia $(\mathrm{Hgb}<11 \mathrm{~g} / \mathrm{dl})$, neutropenia (WBC $<2000$ cells $/ \mathrm{mm}^{3}$ ) and thrombocytopenia $\left(\mathrm{PLT}<125 \times 10^{3}\right.$ cells $\left./ \mathrm{mm}^{3}\right)$ in the study participants was $26.2 \%, 1.6 \%$ and $5.9 \%$ respectively. Among anaemic patients, $62.3 \%, 25.7 \%$ and $12 \%$ had mild, moderate and severe anaemia respectively. Anaemia was significantly higher in patients from the NW region $(p=0.003)$ when compared to those from SW region. Similarly, females $(\mathrm{p}<0.001)$ and HIV/TB co-infected patients $(\mathrm{p}<0.001)$ were more anaemic when compared to males and HIV-mono-infected patients respectively (Figure 2). There was no significant difference $(p=0.959)$ in the prevalence of anaemia between patients on AZT-containing regimen $(24.9 \%)$ compared with those not on AZT regimen (25.1\%).

\section{Virologic responses}

Of the 951 participants, viral load was performed in $89.3 \%$ $(849 / 951)$ and detected in $59.2 \%$, while $40.8 \%$ of the participants had undetectable viral loads (< 40 RNA copies/mL) at median treatment duration of 24 months (Range: 1-156 months). The difference in mean viral load was insignificant $(\mathrm{P}>0.05)$ between participants from the NW and SW regions (Table 2), HIV mono-infected and HIV/ TB co-infected patients and in patients on EFV-based and NVP-based regimens (Table 3). However, the mean viral load was significantly different in the various age groups $(\mathrm{p}=0.033)$ with the highest mean load in those 18-29 years of age (Table 2). The prevalence of virologic failure was $23.2 \%$ at median treatment duration of 16 months (Range: 6 - 132 months), while $76.8 \%$ attained adequate viral suppression at median treatment duration of 28.5 months (Range: 6 - 156 months). Definite virologic failure (> 10,000 RNA copies/mL) was $12.3 \%$ at median treatment duration of 16 months (Range: 6-132 months). Definite virologic failure was significantly prominent in the age group 18-29 years $(18.5 \%, \mathrm{p}=0.013)$, in HIV/TB coinfected patients $(24.5 \%, \mathrm{p}<0.001)$ and among participants from the NW region (20\%, $\mathrm{p}=0.013$ ) (Figure 3 ).

Haemoglobin concentration $(r=0.208, p<0.001), R B C$ $(\mathrm{r}=0.129, \mathrm{p}=0.009)$ and WBC $(\mathrm{r}=0.168, \mathrm{p}=0.001)$ counts correlated significantly with CD4 + T-cell counts. Both haematologic and CD $4+\mathrm{T}$-cell counts showed a negative correlation with viral load although not statistically significant $(\mathrm{p}>0.05)$. Generally, mean $\mathrm{Hgb}$ and 
Table 1 Baseline characteristics of study participants categorized by gender

\begin{tabular}{ccccc}
\hline Variables Category & Overall & Male & Female & P-value \\
& $n(\%)$ & $n(\%)$ & $n(\%)$ & \\
\hline
\end{tabular}

Socio-demographic

Age group (year):

$18-29$

$30-45$

$>45$

Marital status:

Currently

Never

Previously

Income level (XAF):

$\leq 50,000^{*}$

$>50,000$

$\begin{array}{llll}153(16.1) & 19(12.4) & 134(87.6) & <0.001 \\ 561(59.0) & 144(25.7) & 417(74.3) \\ 237(24.9) & 84(35.4) & 153(64.6)\end{array}$

Level of education:

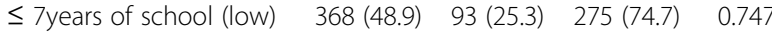

$>7$ years of school (high) $384(51.1) \quad 101(26.3) \quad 283(73.7)$

Region of residence:

South West

$788(82.9) \quad 198(25.1) \quad 590(74.9) \quad 0.191$

North West

$163(17.1) \quad 49(30.1) \quad 144(69.9)$

Management Facility:

AMU

ATC

$111(11.7) \quad 33(29.7) \quad 78(70.3) \quad 0.337$

$840(88.3) \quad 214(25.5) \quad 626(74.5)$

Behavioural

Smoking:

Yes

No

Alcohol use:

Yes

No

Hazardous alcohol use ${ }^{\phi}$

$71(9.1) \quad 48(67.6) \quad 23(32.4) \quad<0.001$

708 (90.9) $151(21.3) \quad 557(78.7)$

Standard alcohol use

Alcoholic-smoker:

Both

Drinks or smokes

\section{Clinical history}

TB co-infection:

Yes

No

Regimen base:

NVP

EFV

\section{$68(16.0) \quad 46(67.6) \quad 22(32.4)$ \\ $358(84.0) \quad 82(22.9) \quad 276(77.1)$}

$423(54.3) \quad 126(29.8) \quad 297(70.2) \quad 0.003$

$356(45.7) \quad 73(20.5) \quad 283(79.5)$

$204(48.2) \quad 61(29.9) \quad 143(70.1)$

0.960

$219(51.8) \quad 65(29.7) \quad 154(70.3)$

$90(10.8) \quad 27(30.0) \quad 63(70.0) \quad 0.354$

$746(89.2) \quad 190(25.5) \quad 556(74.5)$

$614(72.4) \quad 145(23.6) \quad 469(76.4) \quad 0.044$

$234(27.6) \quad 71(30.3) \quad 163(69.7)$
Table 1 Baseline characteristics of study participants categorized by gender (Continued)

\begin{tabular}{lllll}
\hline Duration of ART: & & & & \\
$<12$ months & $216(25.2)$ & $57(26.4)$ & $159(73.6)$ & 0.888 \\
$12-36$ months & $390(45.6)$ & $103(26.4)$ & $287(73.6)$ & \\
$>36$ months & $250(29.2)$ & $62(24.8)$ & $288(75.2)$ &
\end{tabular}

SD-standard deviation, XAF- Central African CFA franc, ${ }^{*} 50,000 \mathrm{XAF}=\sim 100$ US dollars, AMU-approved management unit, ATC-Approved treatment centre, ${ }^{\phi}$ Hazardous alcohol use (Female $<3$ and male $<4$ bottles of beer/occasion), EFV-Efavirenz, NVP-Nevirapine.

CD4 counts improved with ART duration, while mean viral load decreased.

\section{Factors associated with virologic failure}

In a univariate analysis, socio-demographic (Table 4) and behavioural variables (Table 5) except age did not have any significant influence on the prevalence of virologic failure. Failure was associated with the age groups 18-28 years (OR 2.81, 95\% CI: $1.55-5.10$ ) and 30-45 years (OR 1.95, 95\% CI: 1.21 - 3.13). Similarly, patients from the NW region had a higher odds (OR 1.79, 95\% CI: 1.16 - 2.76) of experiencing virologic failure than those from SW region. Distance from treatment centres was also an important determinant of virologic failure. Patients living out of the municipality were more likely to experience virologic failure (OR 2.30, 95\% CI: 1.53 - 3.46) than their counterparts residing within the municipality (Table 4 ).

Similarly, duration of ART, co-infection with TB, CD4 + T-cell count and anaemic status were associated with virologic failure. HIV/TB co-infected patients had remarkably higher odds (OR 3.24, 95\% CI: $1.80-5.84$ ) of experiencing virologic failure than HIV mono-infected patients, while patients on ART for $<12$ months had higher odds (OR 4.24, 95\% CI 2.49 - 7.23) than those who have been on ART for 12-36 months and $>36$ months. Similarly, anaemic patients (OR 2.48, 95\% CI: $1.52-4.07$ ) and patients with CD4+ T-cell count $<200$ cells $/ \mu \mathrm{L}$ (OR 2.49, 95\% CI: 1.0 - 6.31) were also prone to failing ART. Nonetheless, there was no significant difference in virologic failure between those on NVP-based and EFV-based regimen (Table 5).

In a multivariate analysis, involving all significantly associated variables except region of residence, the age group $18-29$ years (AOR 0.27, 95\% CI: $0.10-0.83$ ), coinfection with TB (AOR 0.20, 95\% CI: 0.04- 0.70) and anaemic patients (AOR 2.30, 95\% CI: 1.10 - 4.83) were again associated with virologic failure. Another model which included all significant variables except HIV/TB co-infection, revealed that the age group $18-29$ years $(\mathrm{p}=0.022)$, anaemia $(\mathrm{p}=0.024)$ and distance from treatment centre $(p=0.011)$ with the same adjusted odd ratios as in the previous model were associated with virologic failure. 
Table 2 Mean CD4 + Tcell count, viral load and haematologic parameters of study participants, grouped by region, age and gender

\begin{tabular}{|c|c|c|c|c|c|c|c|c|c|}
\hline \multirow[b]{2}{*}{ Variables Category } & \multicolumn{2}{|c|}{ CD4+ T-cell count } & \multicolumn{2}{|c|}{$\log _{10}$ viral load } & \multicolumn{5}{|c|}{ Mean haematologic parameters \pm SD } \\
\hline & $\mathbf{N}$ & $\begin{array}{c}\text { Mean } \pm \text { SD } \\
{[\text { cells } / \mu \mathrm{L}]}\end{array}$ & $\mathbf{N}$ & $\begin{array}{l}\text { Mean } \pm \text { SD } \\
\text { [copies } / \mathrm{mL}]\end{array}$ & $\mathbf{N}$ & $\begin{array}{l}\mathrm{Hgb} \\
{[\mathrm{g} / \mathrm{dL}]}\end{array}$ & $\begin{array}{c}\text { RBC } \\
{\left[\times 10^{6} / \mathrm{mm}^{3}\right]}\end{array}$ & $\begin{array}{c}\text { WBC } \\
{\left[\times 10^{3} / \mathrm{mm}^{3}\right]}\end{array}$ & $\begin{array}{c}\text { Platelet } \\
{\left[\times 10^{5} / \mathrm{mm}^{3}\right]}\end{array}$ \\
\hline \multicolumn{10}{|l|}{ Region: } \\
\hline Northwest & 59 & $174.9 \pm 120.1$ & 75 & $3.32 \pm 1.38$ & 99 & $11.69 \pm 2.21$ & $3.42 \pm 0.61$ & $4.24 \pm 1.61$ & $2.25 \pm 1.05$ \\
\hline Southwest & 420 & $266.7 \pm 213.8$ & 273 & $3.13 \pm 1.31$ & 538 & $12.07 \pm 2.00$ & $3.69 \pm 0.62$ & $4.41 \pm 1.63$ & $2.36 \pm 0.77$ \\
\hline p-value & & 0.001 & & 0.275 & & 0.094 & $<0.001$ & 0.342 & 0.199 \\
\hline \multicolumn{10}{|l|}{ Age groups (years) } \\
\hline $18-29$ & 74 & $200.1 \pm 154.8$ & 75 & $3.35 \pm 1.33^{\star}$ & 87 & $11.42 \pm 1.69^{\dagger}$ & $3.62 \pm 0.53$ & $3.99 \pm 1.31^{\ddagger}$ & $2.40 \pm 0.88$ \\
\hline $30-45$ & 278 & $256.8 \pm 198.7^{\phi}$ & 195 & $3.23 \pm 1.34^{\star}$ & 371 & $12.09 \pm 2.14$ & $3.69 \pm 0.66$ & $4.44 \pm 1.79$ & $2.35 \pm 0.78$ \\
\hline$>45$ & 127 & $285.8 \pm 242.3^{\phi}$ & 78 & $2.84 \pm 1.24$ & 179 & $12.15 \pm 1.94$ & $3.58 \pm 0.60$ & $4.45 \pm 1.36$ & $2.32 \pm 0.86$ \\
\hline p-value & & 0.018 & & 0.033 & & 0.012 & 0.124 & 0.049 & 0.732 \\
\hline \multicolumn{10}{|l|}{ Gender } \\
\hline Male & 125 & $241.9 \pm 177.9$ & 98 & $3.13 \pm 1.37$ & 158 & $13.24 \pm 2.16$ & $3.93 \pm 0.72$ & $4.39 \pm 1.55$ & $2.20 \pm 0.76$ \\
\hline Female & 354 & $260.1 \pm 215.9$ & 250 & $3.19 \pm 1.31$ & 479 & $11.61 \pm 1.83$ & $3.56 \pm 0.57$ & $4.39 \pm 1.65$ & $2.39 \pm 0.83$ \\
\hline$p$-value & & 0.397 & & 0.724 & & $<0.001$ & $<0.001$ & 0.996 & 0.010 \\
\hline
\end{tabular}

*Higher than 30-45 and $>45$ years [p $<0.025],{ }^{\phi}$ higher than 18-29 years [p $\left.<0.037\right],{ }^{\S}$ higher than $<12$ months [p=0.03], ${ }^{*}$ higher than $<12$ months and

$12-36$ months $[p<0.001]$, ${ }^{\dagger}$ lower than $30-45$ years and $>45$ years $[p<0.005],{ }^{\ddagger}$ lower than $30-45$ and $>45$ years $[p<0.03]$.

\section{Discussion}

Diminution of CD4 + T-cells and haematological complications are hallmarks of HIV disease progression [15] associated with increase morbidity and mortality [16]. The incidence and severity of these factors generally correlate with the stage of the disease with anaemia being the most common and important haematologic predictor of HIV progression to AIDS [17]. In combination with other clinical markers, immuno-haematologic parameters are used routinely in the evaluation and monitoring of HIV-infected persons especially in resource limited settings. They are relatively reliable indicators of prognosis that complement the viral load assay and therefore guide therapeutic decisions regarding antiretroviral treatment [18]. On the other hand, peak viral levels in adults are not predictive of the rate of disease progression

Table 3 Mean CD4 + Tcell count, viral load and haematologic parameters of study participants grouped by ART duration, regimen type and co-infection status

\begin{tabular}{|c|c|c|c|c|c|c|c|c|c|}
\hline \multirow[b]{2}{*}{ Variables Category } & \multicolumn{2}{|c|}{ CD4+ T-cell count } & \multicolumn{2}{|c|}{$\log _{10}$ viral load } & \multicolumn{5}{|c|}{ Mean haematologic parameters \pm SD } \\
\hline & $\mathbf{N}$ & $\begin{array}{c}\text { Mean } \pm \text { SD } \\
{[\text { cell/ } / \mu \mathrm{L}]}\end{array}$ & $\mathbf{N}$ & $\begin{array}{l}\text { Mean } \pm \text { SD } \\
\text { [copies } / \mathrm{mL} \text { ] }\end{array}$ & $\mathbf{N}$ & $\begin{array}{c}\mathrm{Hgb} \\
{[\mathrm{g} / \mathrm{dL}]}\end{array}$ & $\begin{array}{c}\text { RBC } \\
{\left[\times 10^{6} / \mathrm{mm}^{3}\right]}\end{array}$ & $\begin{array}{c}\text { WBC } \\
{\left[\times 10^{3} / \mathrm{mm}^{3}\right]}\end{array}$ & $\begin{array}{c}\begin{array}{c}\text { Platelet } \\
{\left[\times 10^{5} / \mathrm{mm}^{3}\right]}\end{array} \\
\end{array}$ \\
\hline \multicolumn{10}{|c|}{ ART duration (Months) } \\
\hline$<12$ & 106 & $168.9 \pm 143.8$ & 118 & $3.60 \pm 1.27$ & 135 & $11.54 \pm 2.09^{\ell}$ & $3.58 \pm 0.61$ & $4.10 \pm 1.49$ & $2.50 \pm 0.95$ \\
\hline $12-36$ & 255 & $266.3 \pm 214.1^{\S}$ & 153 & $3.07 \pm 1.38$ & 294 & $11.97 \pm 1.90^{\bar{\top}}$ & $3.63 \pm 0.59$ & $4.33 \pm 1.61$ & $2.29 \pm 0.77$ \\
\hline$>36$ & 130 & $318.7 \pm 220.5^{*}$ & 60 & $2.93 \pm 1.05$ & 192 & $12.56 \pm 2.06$ & $3.78 \pm 0.66^{\circ}$ & $4.69 \pm 1.75^{\delta}$ & $2.36 \pm 0.77$ \\
\hline p-value & & $<0.001$ & & 0.001 & & $<0.001$ & 0.008 & 0.004 & 0.054 \\
\hline \multicolumn{10}{|l|}{ Regimen type } \\
\hline EFV-based & 163 & $241.0 \pm 186.4$ & 84 & $3.20 \pm 1.37$ & 179 & $11.91 \pm 1.96$ & $3.59 \pm 0.59$ & $4.28 \pm 1.65$ & $2.48 \pm 0.97$ \\
\hline NVP-based & 297 & $268.9 \pm 219.8$ & 243 & $3.26 \pm 1.30$ & 436 & $12.12 \pm 2.06$ & $3.69 \pm 0.63$ & $4.44 \pm 1.65$ & $2.31 \pm 0.75$ \\
\hline p-value & & 0.171 & & 0.683 & & 0.241 & 0.087 & 0.287 & 0.024 \\
\hline \multicolumn{10}{|l|}{ Co-Infection status } \\
\hline HIV only & 398 & $274.3 \pm 216.6$ & 255 & $3.18 \pm 1.35$ & 574 & $12.14 \pm 2.02$ & $3.71 \pm 0.62$ & $4.44 \pm 1.67$ & $2.36 \pm 0.76$ \\
\hline $\mathrm{HIV} / \mathrm{TB}$ & 66 & $171.1 \pm 112.1$ & 42 & $3.50 \pm 1.34$ & 63 & $10.89 \pm 1.86$ & $3.09 \pm 0.42$ & $3.89 \pm 1.06$ & $2.20 \pm 1.22$ \\
\hline$p$-value & & $<0.001$ & & 0.166 & & $<0.001$ & $<0.001$ & 0.011 & 0.154 \\
\hline
\end{tabular}

EFV-Efavirenz, NVP-Nevirapine, ${ }^{\S}$ higher than $<12$ months $\left[p=0.03\right.$ ], ${ }^{*}$ higher than $<12$ months and $12-36$ months [p $\left.<0.001\right],{ }^{\ell}$ lower than $12-36$ and $>36$ months $[p<0.02],{ }^{\top}$ lower than $>36$ months $[p=0.02],{ }^{\infty}$ higher than $<12$ months $[p=0.012],{ }^{\delta}$ higher 12 months $[p=0.004]$. 


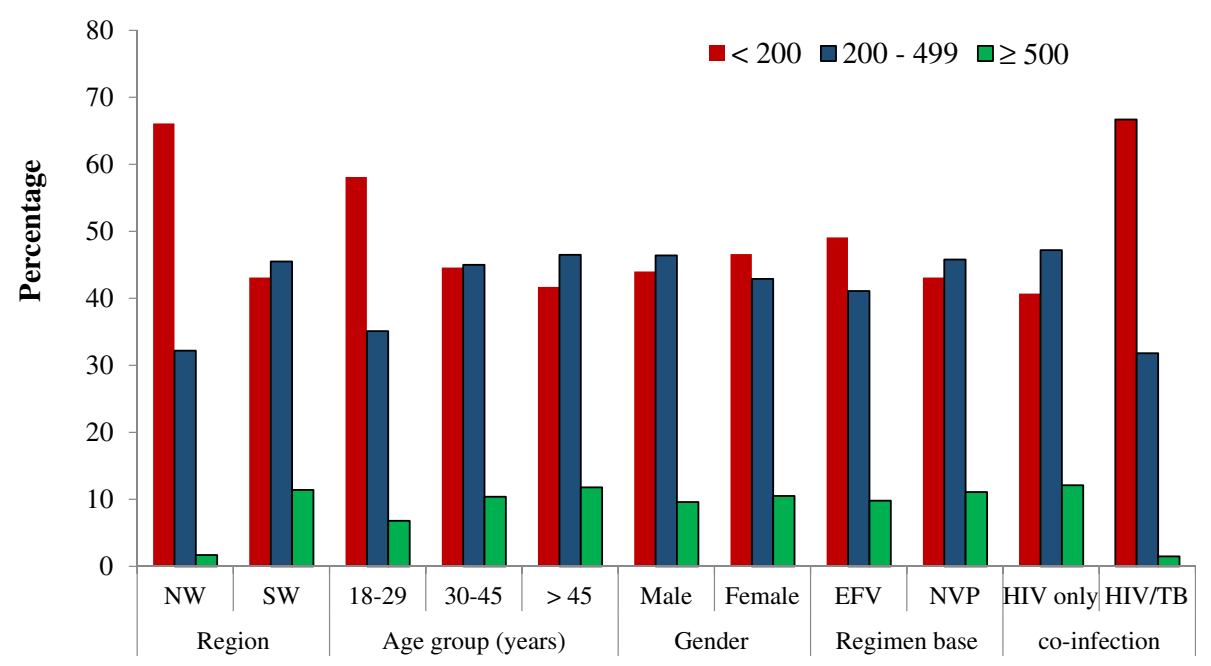

Figure 1 Prevalence of immunologic categories among study participants.

per se. However viral set point, which is most likely a measure of the dynamics between the virulence of the virus strain and the ability of the host immune system to contain the virus, is highly predictive of disease progression [19].

In the present study, there was generally, an overall improvement in the means of immunologic and hematologic parameters of study participants with ART duration. The means of all haematologic parameters except that for platelet counts were significantly higher $(\mathrm{p}<0.001)$ in patients who at recruitment were on ART for $>36$ months. This increase in immuno-hematologic parameters corresponded with a significant decrease in mean viral loads in participants as ART duration increased (Table 3).
A rise in CD4 + T-cell counts significantly correlated with increase values of $\mathrm{Hgb}(\mathrm{p}<0.001), \mathrm{RBC}(\mathrm{p}<0.009)$ and WBC $(\mathrm{p}=0.001)$ and these constitute indicators of improved treatment outcome. These findings corroborate previous studies that demonstrated an improvement in the haematological and immunological parameters as well as reduced morbidity and mortality in HIV infected persons who had been on HAART for a long duration $[20,21]$.

There was also a demographic disparity in immunohaematologic parameters. Over $70 \%$ of participants in the current study were females. Previous studies have also reported a high female population as well as the fact that females are infected early in life than males [22],

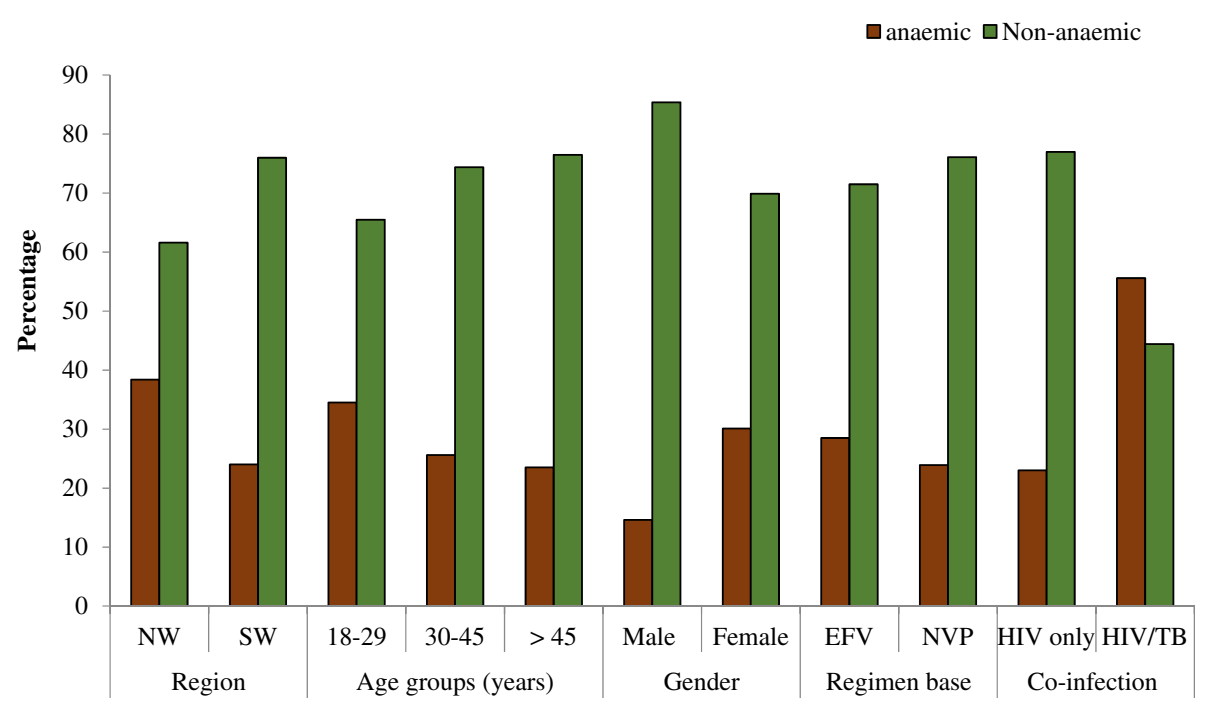

Figure 2 Prevalence of anaemia $[\mathrm{Hgb}<11 \mathrm{~g} / \mathrm{dl}]$ among study participants. 


\section{Viral Load [copies'mL] \\ $\square<400 \quad \square 400-10,000 \quad \square>10,000$}

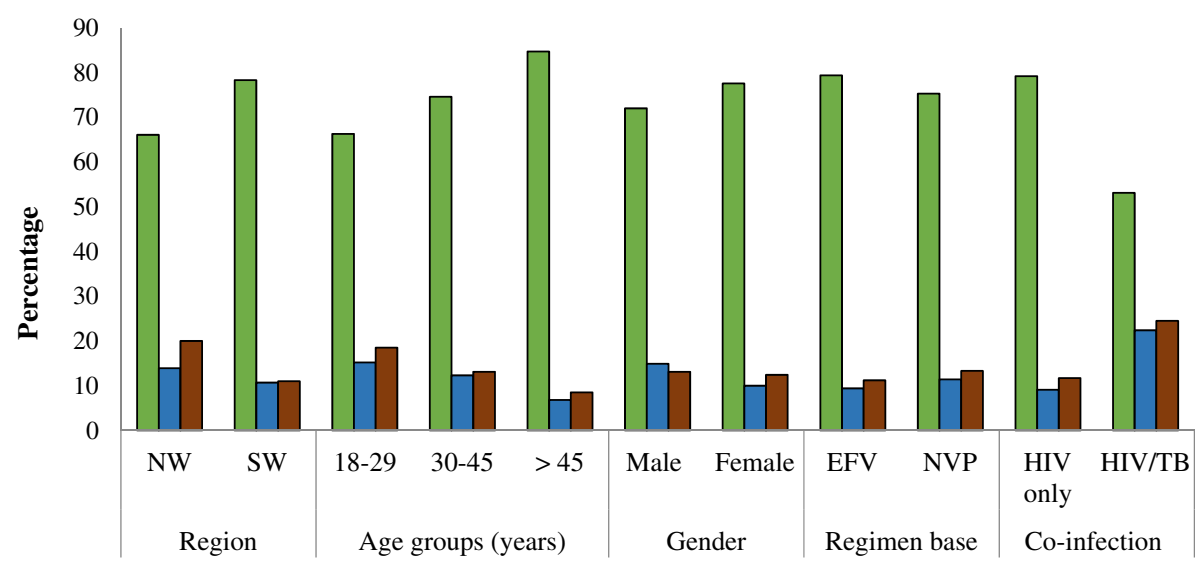

Figure 3 Prevalence of virologic categories among study participants.

Table 4 Demographic and socio-economic factors associated with virologic failure and risk estimates

\begin{tabular}{|c|c|c|c|c|c|c|c|}
\hline \multirow[t]{2}{*}{ Factors Demographic } & \multicolumn{4}{|c|}{ Virologic responses: prevalence, $n(\%)$} & \multicolumn{3}{|c|}{ Risk estimates $(95 \% \mathrm{Cl})$} \\
\hline & $\mathbf{N}$ & VF & AS & p-value & Crude odd ratio & Adjusted odd ratio & p-value \\
\hline \multicolumn{8}{|l|}{ Gender: } \\
\hline Male & 175 & $46(26.3)$ & $129(73.7)$ & 0.260 & $1.26(0.84-1.87)$ & & \\
\hline Female & 502 & $111(22.1)$ & $391(77.9)$ & & 1 & & \\
\hline \multicolumn{8}{|l|}{ Age (years): } \\
\hline $18-29$ & 96 & $31(32.3)$ & $65(67.7)$ & 0.002 & $2.81(1.55-5.10)$ & $0.27(0.10-0.83)$ & 0.024 \\
\hline $30-45$ & 402 & $100(24.9)$ & $302(75.1)$ & & $1.95(1.21-3.13)$ & $0.44(0.20-1.07)$ & 0.079 \\
\hline$>45$ & 179 & $26(14.5)$ & $153(85.5)$ & & 1 & 1 & \\
\hline \multicolumn{8}{|l|}{ Region: } \\
\hline Northwest & 120 & $39(32.5)$ & $81(67.5)$ & 0.008 & $1.79(1.16-2.76)$ & $0.20(0.10-0.74)^{*}$ & \\
\hline Southwest & 557 & $118(21.2)$ & 439 (78.8) & & 1 & 1 & \\
\hline \multicolumn{8}{|l|}{ Distance to TC: } \\
\hline Out of municipality & 281 & $80(28.5)$ & $201(71.5)$ & $<0.001$ & $2.30(1.53-3.46)$ & $0.42(0.20-0.94)$ & 0.011 \\
\hline Within municipality & 312 & $46(14.7)$ & $266(85.3)$ & & 1 & 1 & \\
\hline \multicolumn{8}{|l|}{ Socio-economic } \\
\hline \multicolumn{8}{|l|}{ Level of education } \\
\hline Low ( $\leq 7 y$ rs. of school) & 273 & $46(16.8)$ & $227(83.2)$ & 0.118 & $0.72(0.47-1.09)$ & & \\
\hline High (> 7 yrs. of school) & 309 & $68(22.0)$ & $241(78.0)$ & & 1 & & \\
\hline \multicolumn{8}{|l|}{ Income level: } \\
\hline$\leq 50,000$ XAF $^{d}$ & 480 & $94(19.6)$ & $386(80.4)$ & 0.960 & $0.99(0.58-1.69)$ & & \\
\hline$>50,000 \times A F$ & 101 & 20 (19.8) & $81(80.2)$ & & 1 & & \\
\hline \multicolumn{8}{|l|}{ Marital status: } \\
\hline Never married & 167 & $45(25.7)$ & $124(74.3)$ & & $1.57(0.92-2.69)$ & & \\
\hline Currently married & 245 & $42(17.1)$ & $203(82.9)$ & 0.079 & $0.94(0.55-1.59)$ & & \\
\hline Previously married & 155 & $28(18.1)$ & $127(81.9)$ & & 1 & & \\
\hline
\end{tabular}

"Model including region of residence and excluding HIV/TB co-infection, VF: Virologic failure (viral load $>400$ RNA copies/mL after 6 months of ART), AD: Adequate suppression ( $\leq 400 \mathrm{RNA}$ copies $/ \mathrm{mL}$ after 6 months of treatment), TC: treatment centre, Cl- confidence interval, ${ }^{d} 50,000$ XAF $=\sim 100$ US dollars. 
Table 5 Behavioural and clinical factors associated with virologic failure and risk estimates

\begin{tabular}{|c|c|c|c|c|c|c|c|}
\hline \multirow[t]{2}{*}{ Factors Behavioural } & \multicolumn{4}{|c|}{ Virologic responses: prevalence, $n$ (\%) } & \multicolumn{3}{|c|}{ Risk estimates $(95 \% \mathrm{Cl})$} \\
\hline & $\mathbf{N}$ & VF & AS & p-value & Crude odd ratio & Adjusted odd ratio & p-value \\
\hline \multicolumn{8}{|l|}{ Alcohol: } \\
\hline Yes & 313 & $65(20.8)$ & $248(79.2)$ & 0.927 & $0.98(0.66-1.46)$ & & \\
\hline No & 280 & $59(21.1)$ & $221(78.9)$ & & 1 & & \\
\hline \multicolumn{8}{|l|}{ Smoking: } \\
\hline Yes & 55 & $15(27.3)$ & $40(72.7)$ & 0.223 & $1.48(0.79-2.77)$ & & \\
\hline No & 538 & $109(20.3)$ & $429(79.7)$ & & 1 & & \\
\hline \multicolumn{8}{|l|}{ Clinical history } \\
\hline \multicolumn{8}{|l|}{ Duration of ART } \\
\hline (months): $\leq 12$ & 108 & $48(44.4)$ & $60(55.6)$ & $<0.001$ & $4.24(2.49-7.23)$ & $1.11(0.40-3.16)$ & 0.851 \\
\hline $12-36$ & 361 & $76(21.1)$ & $285(78.9)$ & & $1.41(0.90-2.22)$ & $1.57(0.73-3.36)$ & 0.251 \\
\hline$>36$ & 208 & $33(15.9)$ & $175(84.1)$ & & 1 & 1 & \\
\hline \multicolumn{8}{|l|}{ Regimen type: } \\
\hline EFV-based & 177 & 35 (19.8) & $142(80.2)$ & 0.232 & $0.77(0.51-1.18)$ & & \\
\hline NVP-based & 492 & $119(24.2)$ & $373(75.8)$ & & 1 & & \\
\hline \multicolumn{8}{|l|}{ Co-infection status: } \\
\hline HIV/TB co-infected & 51 & $22(45.1)$ & $28(54.9)$ & $<0.001$ & $3.24(1.80-5.84)$ & $0.20(0.04-0.70)$ & 0.014 \\
\hline HIV only & 589 & $119(20.2)$ & $470(79.8)$ & & 1 & 1 & \\
\hline \multicolumn{8}{|l|}{ CD4+T-cell (cells $/ \mu \mathrm{L}):$} \\
\hline$<200$ & 154 & $46(29.9)$ & $108(70.1)$ & 0.002 & $2.49(1.0-6.31)$ & $0.68(0.23-2.05)$ & 0.497 \\
\hline $200-499$ & 165 & $24(14.5)$ & $141(85.5)$ & & $1.69(0.63-4.49)$ & $1.22(0.40-3.75)$ & 0.724 \\
\hline$\geq 500$ & 41 & $06(14.6)$ & $35(85.4)$ & & 1 & 1 & \\
\hline \multicolumn{8}{|l|}{ Anaemic status $(\mathrm{g} / \mathrm{dL})$ : } \\
\hline Anaemic & 114 & $34(29.8)$ & $80(70.2)$ & $<0.001$ & $2.48(1.52-4.07)$ & $2.30(1.10-4.83)$ & 0.028 \\
\hline Non-anaemic & 376 & $55(14.6)$ & $321(85.4)$ & & 1 & 1 & \\
\hline
\end{tabular}

[23]. Females have also been observed to have a better health-seeking attitude than males [24]. Our results show that increase in age had a better prediction in terms of immuno-haematologic and virologic responses to ART. Furthermore, the mean CD $4+$ T-cell count of HIV/TB co-infected patients was 1.6 times lower than that of HIV mono-infected persons. Co-infected patients were also more anaemic than HIV mono-infected patients, which is in line with a previous report by Kufa et al. [25]. CD4 + T-cell-count of $<200$ cells $/ \mu \mathrm{L}$ was predominant among HIV/TB co-infected patients (Figure 1). In a previous study, CD $4+\mathrm{T}$-cell count $<200$ cells $/ \mu \mathrm{L}$ was associated with an increased risk of anaemia [26].

Mean viral load and CD4 + T-cell counts did not differ between males and females, although prior studies $[27,28]$ suggest a significant influence of gender on viral dynamics and the immune response. In a recent report, women showed consistently better immune responses to treatment than did men in a virally suppressed population [29]. However, in our study, females were more at risk of developing anaemia compared with males $(\mathrm{p}<0.001)$ which is in line with findings by Levine et al. [30] who attributed this difference to sex and race. Similar findings by Volberding et al. [31] attributed anaemia to menstrual blood loss in women and to the drain on iron stores that occur with pregnancy and delivery. Even with the use of HAART, anaemia remains strongly and consistently associated with HIV disease progression [32]. Although the prevalence of severe anaemia has decreased since the introduction of HAART, mild-to-moderate anaemia continues to be common [33]. In this study, $61.3 \%$, $26.9 \%$ and $11.8 \%$ of anaemic patients suffered from mild, moderate and severe anaemia respectively. Our study did not show any significant difference in the occurrence of anaemia between patients on zidovudine-containing regimens and those without as also reported by Semba et al. [34].

In line with previous reports our results further indicated that there was no significant difference in mean CD4 count [35] and mean viral load [36,37] in those 
treated with NVP-based and EFV-based regimens, in contrast to another report [38] that showed a better outcome with Efavirenz-based ART compared with Nevirapine. The lower mean immuno-hematologic parameters seen in participants from the NW when compared with those from the SW region was probably because most of the HIV/TB co-infected patients were from the NW region. Moreover, our results showed HIV co-infection with TB was associated with poor immunologic and hematologic responses.

Attaining and maintaining viral load to an undetectable level is the key to mitigating long-term risk of AIDSdefining illnesses among HIV-positive patients with low CD4 counts. Patients who are initiated on ART and experience rapid CD4 increase, have a lower risk of AIDS illness than those with a slow response. Nonetheless, this difference diminishes after six months provided that viral load remains undetectable [39]. At a median treatment duration of 24 months, $40.8 \%$ of the study participants had undetectable viral loads with an overall $76.8 \%$ adequate viral suppression rate at a median treatment duration of 28.5 months. The prevalence of virologic failure was $23.2 \%$ of which $12.3 \%$ reflected a definite virologic failure. These rates are similar to those reported in other resource-limited settings [40] and comparable to those reported in developed countries. In spite of earlier incertitude, this shows that ART programs based on the public health approach using similar regimens in resource-limited countries, perform as effective as those seen in clinical cohorts in developed countries [41-43].

Even though socioeconomic status has been associated with HIV treatment outcomes [44] our study surprisingly did not suggest an association between socio-economic or behavioural predictors of virologic failure. However, participants from the NW region, and patients residing far from treatment centres were more likely to experience virologic failure. Previous studies and extensive reviews on barriers to accessing HIV treatment and negative treatment outcomes suggest that longer distances from treatment sites are associated with poorer outcomes $[45,46]$.

The higher occurrence of virologic failure in the younger age group of 18-29 years corroborates a previous report by Anude et al. [47] and this can be attributed mainly to nonadherence to treatment among the youths [46]. Other studies have also demonstrated that improved ART outcomes increases with age [48]. Similarly, virologic failure was common among HIV/TB co-infected and anaemic patients as well as in patients with CD4 + T-cell counts of $<200$ cell $/ \mu \mathrm{L}$. Studies conducted by Skowron et al. [49] demonstrated that CD4 + T-cell count is a better predictor of viral suppression. Likewise, Bello et al. [50] showed that TB co-morbidity had a significant influence on the occurrence of virologic failure by accelerating the course of HIV induced disease resulting from CD + T-cells decline.

\section{Conclusions}

This study demonstrates a favourable immuno-haematologic and virologic outcome following antiretroviral therapy. Anaemia, co-infection with TB, the age group 18-29 years and distance from the treatment centre independently predicted virologic failure. Despite this favourable outcome, it is imperative for the National AIDS Control Committee (NACC) to step-up efforts to improve on ARV distribution, proper assessment and management of anaemia, early diagnosis and treatment of $\mathrm{TB}$ and treatment adherence counselling especially in younger patients.

\section{Additional file}

Additional file 1: Multilingual abstracts in the six official working languages of the United Nations.

\section{Abbreviations}

ART: Antiretroviral therapy; HAART: Highly active antiretroviral therapy; ARV: Antiretroviral; AZT: Zidovudine; NVP: Nevirapine; EFV: Efavirenz; 3TC: Stavudine; SW: Southwest; NW: Northwest; NACC: National AIDS control committee.

\section{Competing interests}

The authors declare that they have no competing interests.

\section{Authors' contributions}

Conceived and designed the experiments: HDM, TN-A and FC-N. Sample collection and Laboratory analysis: HDM, KAT, MHA and BAN. Analysed the data: HDM and PNA. Contributed reagents/materials: HDM, TN-A, and FC-N. Wrote the paper: HDM, PNA, FC-N and TNA. All authors read and approved the final manuscript

\section{Acknowledgement}

We would like to acknowledge and thank immensely all participants and staff of the HIV treatment centres/management units where this study was conducted. BioCollections Worldwide Inc., Miami, Florida, USA, provided financial support and performed viral load analysis. Financial assistance was also received from the University of Buea 2009-2010 Research Grant awarded to Professor Theresa Nkuo-Akenji. The content of this paper is exclusively the responsibility of the authors and does not necessarily represent the official views of the funding institutions.

\section{Author details}

'Department of Microbiology and Parasitology, University of Buea, P.O. Box 63, Buea, Cameroon. 'BioCollections Worldwide Inc. Regional Office, Buea, Cameroon. ${ }^{3}$ Regional Delegation of Public Health, Southwest Region, Cameroon. ${ }^{4}$ Faculty of Science Clinical Diagnostic Laboratory, University of Buea, Buea, Cameroon

Received: 10 July 2013 Accepted: 27 January 2014

Published: 30 January 2014

\section{References}

1. Mills E, Nachega J, Buchan I: Adherence to antiretroviral therapy in sub-Saharan Africa and North America. J Am Med Assoc 2006, 296:679-90.

2. Crowe S, Turnbull S, Oelrichs R, Dunne A: Monitoring of human immunodeficiency virus infection in resource-constrained countries. Clin Infect Dis 2003, 37:S25-35.

3. Metzner KJ, Binley JM, Gettie A, Marx P, Nixon DF, Connor Rl: Tenofovir treatment augments anti-viral immunity against drug-resistant SIV challenge in chronically infected rhesus macaques. Retrovirology 2006, 3:97.

4. Omoregie R, Omokaro EU, Palmer O, Ogefere HO, Egbeobauwaye A, Adeghe JE, Osakue SI, Ihemeje V: Prevalence of anaemia among HIV-infected patients in Benin City. Nigeria. Tanzan. J. Health Res. 2009, 11:1-4. 
5. Gallant JE: Strategies for long-term success in the treatment of HIV infection. J Am Med Assoc 2000, 283:1329-34.

6. Haubrich $\mathrm{R}$, Currier J: A randomized study of the utility of human immunodeficiency virus RNA measurement for the management of antiretroviral therapy. Clin Infect Dis 2001, 33:1060-1068.

7. WHO: Antiretroviral Therapy for HIV infection in Adults and Adolescents: Recommendations for a public health approach 2006 revision. HIV/AIDS Programme: Strengthening health services to fight HIV/AIDS. Geneva: World Health Organization; 2006.

8. Apondi R, Bunnell R, Ekwaru JP, Moore D, Bechange S, Khana K, King R, Campbell J, Tappero J, Mermin J: Sexual behavior and HIV transmission risk of Ugandan adults taking antiretroviral therapy: 3 year follow-up. AIDS 2011, 25:1317-1327.

9. Institut National de la Statistique, Ministère de l'Économie de la Planification et de l'Aménagement du Territoire et Ministère de la Santé Publique: Enquête Démographique et de Santé et à Indicateurs Multiples (EDS-MICS):2011. Yaoundé: ICF International Calverton, Maryland; 2012

10. BUCREP: 3ème Recensement Général de la population et de l'Habitat (3e RGPH): La Population du Cameroun en 2010. Yaoundé: Bureau Central des Recensements et des Etudes de la Population; 2010.

11. National AIDS Control Committe- Central Technical Group (NACC-CTG): The Impact of HIV and AIDS in Cameroon through 2020. 2010.

12. Ramadhani HO, Thielman NM, Landman KZ, Ndosi EM, Gao F, Kirchherr JL, Shah R, Shao HJ, Morpeth SC, Mcneill JD, Shao JF, Bartlett JA, Crump JA: Predictors of Incomplete Adherence, Virologic Failure, and Antiviral Drug Resistance among HIV-Infected Adults Receiving Antiretroviral Therapy in Tanzania. Clin Infect Dis 2007, 45:1492-1498.

13. WHO: Haemoglobin concentrations for the diagnosis of anaemia and assessment of severity. Micronutrients Indicators: Vitamin and Mineral Nutrition Information System. Geneva: World Health Organization; 2011.

14. Tsuchiya N, Pathipvanich P, Yasuda T, Mukoyama Y, Rojanawiwat A, Matsubayashi T, Saeng-aroon S, Auwanit W, Matsuyama A, Sawanpanyalert P, Ariyoshi K, Hospital L: Demographic, Socio-economic, Behavioural and Clinical Factors Predicting Virologic Failure with Generic Fixed-Dose Combination Antiretroviral Therapy before Universal Health Insurance Coverage in Northern Thailand. Southeast Asian I Trop Med Public Health 2009, 40:71-82.

15. Shearer WT, Rosenblatt HM, Schluchter MD, Mofenson LM, Denny TN: Immunologic targets of HIV infection: T cells. NICHD IVIG Clinical Trial Group, and the NHLBI P2C2 Pediatric Pulmonary and Cardiac Complications of HIV Infection Study Group. Ann N Y Acad Sci 1993, 693:35-51.

16. Cosby CD: Hematologic Disorders Associated with Human Immunodeficiency Virus and AIDS. J Infus Nurs 2007, 30(1):22-32

17. Obirikorang C, Yeboah F: Blood haemoglobin measurement as a predictive indicator for the progression of HIV/AIDS in resource-limited setting. J Biomed Sci 2009, 16:102

18. Kilaru KR, Kumar A, Sippy N: CD4 cell counts in adults with newly diagnosed HIV infection in Barbados. Pan Am. J. Public Heal. 2004, 16:302-7.

19. Richardson BA, Mbori-ngacha D, John-stewart GC, Nduati R, Dana D, Emery S, Kreiss JK, Lavreys L, Panteleeff DD, Overbaugh J: Comparison of Human Immunodeficiency Virus Type 1 Viral Loads in Kenyan Women, Men, and Infants during Primary and Early Infection Comparison of Human Immunodeficiency Virus Type 1 Viral Loads in Kenyan Women, Men, and Infants during Primary and Early. J Virol 2003, 77:7120-7123

20. Wachukwu CK, Abbey SD, Busuri Fl, Erhabor O: Changes in Immunohaematological Characteristics of Human Immunodeficiency. J. Med. Lab. Sci. 2007, 13:35348.

21. Owiredu WKBA, Quaye L, Amidu NA-MO: Prevalence of anaemia and immunological markers among Ghanaian HAART-naïve HIV-patients and those on HAART. Afr Health Sci 2011, 11:2-15.

22. Magadi MA: Understanding the gender disparity in HIV infection across countries in sub-Saharan Africa: evidence from the Demographic and Health Surveys. Sociol. Heal. IIIn. 2011, 33:522-539.

23. Amornkul PN, Vandenhoudt $H$, Nasokho P, Odhiambo F, Mwaengo D, Hightower A, Buvé A, Misore A, Vulule J, Vitek C, Glynn J, Greenberg A, Slutsker L, De Cock KM: HIV prevalence and associated risk factors among individuals aged 13-34 years in Rural Western Kenya. PLoS One 2009, 4:e6470.
24. Saathoff E, Villamor E, Mugusi F, Bosch RJ, Urassa W, Fawzi WW: Anemia in adults with tuberculosis is associated with HIV and anthropometric status in Dar es Salaam, Tanzania. Int. J. Tuberc. Lung Dis. 2013, 15:925-932.

25. Kufa T, Mngomezulu V, Charalambous S, Hanifa Y, Fielding K, Grant AD, Wada N, Chaisson RE, Churchyard GJ, Gounder CR: Undiagnosed tuberculosis among HIV clinic attendees: association with antiretroviral therapy and implications for intensified case finding, isoniazid preventive therapy, and infection control. J Acquir Immune Defic Syndr 2012, 60:e22-8.

26. Mata-Marín JA, Gaytán-Martínez JE, Martínez-Martínez RE, Arroyo-Anduiza Cl, Fuentes-Allen JL, Casarrubias-Ramirez M: Risk factors and correlates for anemia in HIV treatment-naïve infected patients: a cross-sectional analytical study. BMC Res. Notes 2010, 3:230.

27. Farzadegan H, Hoover DR, Astemborski J, Lyles CM, Margolick JB, Markham RB, Quinn TC, Vlahov D: Early reports Sex differences in HIV-1 viral load and progression to AIDS. Lancet 1998, 352:1510-14.

28. Ballesteros-Zebadúa P, Villarreal C, Cocho G, Huerta L, Estrada JL: Differences in HIV-1 viral loads between male and female antiretroviraluntreated mexican patients. Arch Med Res 2013, 4409:3-4.

29. Maskew M, At B, Westreich D, Mcnamara L, Ap M, Mp F: Gender differences in mortality and CD4 count response among virally suppressed HIV-positive patients. J. Womens Heal. 2013, 22:1-2.

30. Levine A, Berhane K: Prevalence and correlates of anemia in a large cohort of HIV-infected women: Women's Interagency HIV Study. J Acquir Immune Defic Syndr 2001, 26:28-35.

31. Volberding PA, Levine AM, Dieterich D, Mildvan D, Mitsuyasu R, Saag M: Anemia in HIV infection: clinical impact and evidence-based management strategies. Clin Infect Dis 2004, 38:1454-63.

32. Mocroft A, Kirk O, Barton SE, Dietrich $M$, Proenca R, Colebunders $B$ Pradier C, DArminio Monforte A, Ledergerber B, Lundgren JD: Anaemia is an independent predictive marker for clinical prognosis in HIV-infected patients from across Europe. EuroSIDA study group. AIDS 1999, 13:943-50.

33. Moore R, Forney D: Anemia in HIV-infected patients receiving highly active antiretroviral therapy. J Acquir Immune Defic Syndr 2002, 29:54-57.

34. Semba RD, Shah N, Klein RS, Mayer KH, Schuman P, Vlahov D: Prevalence and cumulative incidence of and risk factors for anemia in a multicenter cohort study of human immunodeficiency virus-infected and -uninfected women. Clin Infect Dis 2002, 34:260-266.

35. Patel AK, Pujari S, Patel K, Patel J, Shah N, Patel B, Gupte N: Nevirapine Versus Efavirenz Based Antiretroviral Treatment in Naïve Indian Patients: comparison of comparison of effectiveness in clinical cohort. J Assoc Physicians India 2006, 54:915-918.

36. Núnez M, Soriano V, Martín-Carbonero L, Barrios A, Barreiro P, Blanco F, García-Benayas T, González-Lahoz J: SENC (Spanish efavirenz vs. nevirapine comparison) trial: a randomized, open-label study in HIV-infected naive individuals. HIV Clin. Trials 2002, 3:186-94.

37. Van den Berg-Wolf M, Hullsiek KH, Peng G, Kozal MJ, Novak RM, Chen L, Crane LR, Macarthur RD: Virologic, immunologic, clinical, safety, and resistance outcomes from a long-term comparison of efavirenz-based versus nevirapine-based antiretroviral regimens as initial therapy in HIV-1-infected persons. HIV Clin Trials 2008, 9:324-36.

38. McComsey G, Kitch D, Daar E: Inflammation markers after randomization to abacavir/lamivudine or tenofovir/emtricitabine with efavirenz or atazanavir/ritonavir: ACTG A5224 s, A5202 substudy. AIDS 2012, 26:1371-1385

39. Zoufaly A, An der Heiden M, Kollan C, Bogner JR, Fätkenheuer G, Wasmuth JC, Stoll M, Hamouda O, van Lunzen J: Clinical outcome of HIV-infected patients with discordant virological and immunological response to antiretroviral therapy. J Infect Dis 2011, 203:364-71.

40. Arnedo $M$, Alonso $E$, Eisenberg $N$, Ibáñez L, Ferreyra $C$, Jaén $A$, Flevaud L, Khamadi S, Roddy P, Gatell JM, Dalmau D: Monitoring HIV viral load in resource limited settings: still a matter of debate? PLoS One 2012, 7:e47391.

41. Ferradini L, Jeannin A, Pinoges L, Izopet J, Odhiambo D, Mankhambo L, Karungi G, Szumilin E, Balandine S, Fedida G, Carrieri MP, Spire B, Ford N, Tassie J-M, Guerin PJ, Brasher C: Scaling up of highly active antiretroviral therapy in a rural district of Malawi: an effectiveness assessment. Lancet 2006, 367:1335-42.

42. Bartlett JA, Buda JJ, von Scheele B, Mauskopf JA, Davis EA, Elston R, King MS, Lanier ER: Minimizing resistance consequences after virologic failure on initial combination therapy: a systematic overview. J Acquir Immune Defic Syndr 2006, 41:323-31. 
43. Sow P, Otieno L, Bissagnene E: Implementation of an antiretroviral access program for HIV-1-infected individuals in resource-limited settings: clinical results from 4 African countries. J Acquir Immune Defic Syndr 2007, 44:262-267.

44. Koenig S, Leandre F, Farmer P: Scaling-up HIV treatment programmes in resource-limited settings: the rural Haiti experience. AIDS 2004, 18(supl 3):S21-25.

45. Posse M, Meheus F, van Asten $H$, van der Ven A, Baltussen R: Barriers to access to antiretroviral treatment in developing countries: a review. Trop. Med. Int. Heal. 2008, 13:904-13.

46. Charurat M, Oyegunle M, Benjamin R, Habib A, Eze E, Ele P, Ibanga I, Ajayi S, Eng M, Mondal P, Gebi U, Iwu E, Abimiku A, Dakum P, Farley J, Blattner W: Patient retention and adherence to antiretrovirals in a large antiretroviral therapy program in Nigeria: a longitudinal analysis for risk factors. PloS One 2010, 5:e10584

47. Anude CJ, Eze E, Onyegbutulem HC, Charurat M, Etiebet M-A, Ajayi S, Dakum P, Akinwande O, Beyrer C, Abimiku A, Blattner W: Immuno-virologic outcomes and immuno-virologic discordance among adults alive and on anti-retroviral therapy at 12 months in Nigeria. BMC Infect Dis 2013, 13:113.

48. Glass TR, De Geest $\mathrm{S}$, Weber R, Vernazza PL, Rickenbach M, Furrer H, Bernasconi E, Cavassini M, Hirschel B, Battegay M, Bucher HC: Correlates of self-reported nonadherence to antiretroviral therapy in HIV-infected patients: the Swiss HIV Cohort Study. J Acquir Immune Defic Syndr 2006, 41:385-92.

49. Skowron G, Street J, Obee E: Baseline CD4+ cell count, not viral load, correlates with virologic suppression induced by potent antiretroviral therapy. J Acquir Immune Defic Syndr 2001, 28:313-319.

50. Bello EJM, Correia AF, Marins JRP, Merchan-Hamann E, Kanzaki LIB: Predictors of virologic failure in HIV/AIDS patients treated with highly active antiretroviral therapy in Brasília, Brazil during 2002-2008. Drug Target Insights 2011, 5:33-41.

doi:10.1186/2049-9957-3-5

Cite this article as: Meriki et al:: Immuno-haematologic and virologic responses and predictors of virologic failure in HIV-1 infected adults on first-line antiretroviral therapy in Cameroon. Infectious Diseases of Poverty 2014 3:5.

\section{Submit your next manuscript to BioMed Central and take full advantage of:}

- Convenient online submission

- Thorough peer review

- No space constraints or color figure charges

- Immediate publication on acceptance

- Inclusion in PubMed, CAS, Scopus and Google Scholar

- Research which is freely available for redistribution 